\title{
Nonlinear radiation generation processes in the auroral acceleration region
}

\author{
Raymond Pottelette and Matthieu Berthomier \\ Laboratoire de Physique des Plasmas, UPMC, CNRS UMR 7648, 4 place Jussieu, 75252 Paris CEDEX 05, France \\ Correspondence to: Raymond Pottelette (raymond.pottelette@1pp.polytechnique.fr)
}

Received: 19 May 2017 - Revised: 17 October 2017 - Accepted: 18 October 2017 - Published: 22 November 2017

\begin{abstract}
It is known from laboratory plasma experiments that double layers (DLs) radiate in the electromagnetic spectrum; but this is only known qualitatively. In these experiments, it was shown that the electron beam created on the high-potential side of a DL generates nonlinear structures which couple to electromagnetic waves and act as a sender antenna. In the Earth auroral region, observations performed by auroral spacecraft have shown that DLs occur naturally in the source region of intense radio emissions called auroral kilometric radiation (AKR). Very high time-, spatial-, and temporal-resolution measurements are needed in order to characterize waves and particle distributions in the vicinity of DLs, which are moving transient structures. We report observations from the FAST satellite of a localized largeamplitude parallel electric field $\left(\sim 300 \mathrm{mV} \mathrm{m}^{-1}\right)$ recorded at the edges of the auroral density cavity. In agreement with laboratory experiments, on the high-potential side of the DL, elementary radiation events are detected. They occur substantially above the local electron gyrofrequency and are associated with the presence of electron holes. The velocity of these nonlinear structures can be derived from the measurement of the Doppler-shifted AKR frequency spectrum above the electron gyrofrequency. The generated electron holes appear as the nonlinear evolution of electrostatic waves generated by the electron-electron two-stream instability because they propagate at about half the beam velocity. It is pointed out that, in the vicinity of a DL, the shape of the electron distribution gives rise to a significant power recorded in the left-hand polarized ordinary (LO) mode.
\end{abstract}

Keywords. Space plasma physics (radiation processes)

\section{Introduction}

Electromagnetic radiation from double layers (DLs) has been extensively studied in laboratory plasma experiments (Volwerk, 1993; Lindberg, 1993; Brenning et al., 2004). The spectrum was found to contain characteristic peaks around the electron gyrofrequency and electron plasma frequency (Gunell and Löfgren, 1997; Löfgren and Gunell, 1998). It was shown that the electron beam, created on the highpotential side of the DL, generates high-frequency nonlinear structures which couple to electromagnetic waves and act as a sender antenna. These experimental results demonstrated that there is a possibility of very high efficiency in converting electric energy to radiation.

In space, spacecraft observations have revealed that the direct consequence of the electron parallel acceleration processes is that the Earth acts as an intense radio source in the kilometre wavelength range (Benediktov et al., 1965; Gurnett, 1974). This offers the possibility of investigating locally the eventual mechanisms leading to electromagnetic radiation generation in the vicinity of a DL. The cyclotron maser instability is now widely acknowledged as being the most likely mechanism leading to auroral kilometric radiation (AKR) generation (Wu and Lee, 1979; Louarn et al., 1990; Ergun et al., 2000). In the source region identified as the auroral density cavity, an unstable horseshoe electron distribution exhibiting large positive velocity gradients in the direction perpendicular to the local magnetic field provides the free energy for AKR (Ergun et al., 2000; Treumann, 2006). Such a distribution is generated when a localized DL accelerates the electrons earthward that propagate into an increasing magnetic field (Chiu and Schulz, 1978). The parallel accelerated electrons need to travel a long distance (several thousand kilometres) before forming a horseshoe distribution; this implies that in the auroral region the AKR gen- 
eration takes place far away from a DL. Therefore, these observational results disagree with those obtained in laboratory experiments unless another mechanism leading to radiation generation is identified. Accordingly, there is a need to determine what kind of electron distribution is present and how the auroral plasma may radiate in the vicinity of a DL. High time-resolution measurements, both for waves and particles, are required to identify the presence of DLs which are localized moving transient structures. The higher resolution of FAST measurements as compared to previous auroral missions offers the opportunity to investigate the physical processes taking place in the neighbourhood of DLs. These localized parallel electric fields appear to be located at the interface of the auroral density cavity. We present some observations, recorded on the high-potential side of a largeamplitude DL $\left(\sim 300 \mathrm{mV} \mathrm{m}^{-1}\right)$, which highlight the generation of electromagnetic sporadic emissions in association with nonlinear coherent structures (electron holes). These emissions take place substantially $(\sim 10 \%)$ above the local electron gyrofrequency and the associated wave electric fields are partially polarized in a direction parallel to the magnetic field indicating the probable presence of left-hand polarized ordinary (LO)-mode radiation. The measurement of the Doppler-shifted AKR frequency spectrum allows the determination of the electrons hole velocity. It turns out that the velocity range is about half the beam velocity, in fair agreement with the electron-electron two-stream instability. The generated electron holes appear as the nonlinear evolution of electrostatic waves generated by this instability.

\section{Observations}

Figure 1 displays $12 \mathrm{~s}$ of data (horizontal axis) illustrating the crossing of an anti-earthward-directed large-amplitude DL $\left(320 \mathrm{mV} \mathrm{m}^{-1}\right)$ located in the AKR source region. The FAST spacecraft was at invariant latitude $\sim 68^{\circ}$ and altitude $\sim 4150 \mathrm{~km}$ (orbit 1761). As evidenced in the top panels (a) the spacecraft approaches the DL from the low-potential side at $\sim 06: 44: 44$ UT and enters a region of a steep density gradient. Figure $1 \mathrm{~b}$ and $\mathrm{c}$ show the electric field spectral power density as a function of frequency and time. Panel (b) represents the electric field spectral power density measured by the on-board sweep frequency analyser (SFA) as a function of frequency and time. The frequency span is from 0 to $500 \mathrm{kHz}$ at $15 \mathrm{kHz}$ bandwidth. The dark line around $365 \mathrm{kHz}$ is $f_{\mathrm{ce}}$, the local electron cyclotron frequency. The waves recorded near and above $f_{\text {ce }}$ are AKR; at low frequency (less than $\sim 20 \mathrm{kHz}$ ), intense VLF waves can be identified as hiss emissions, which present an upper cut-off near the local plasma frequency $f_{\mathrm{p}}$ (Persoon et al., 1988); the observations imply that $f_{\mathrm{p}} \ll f_{\mathrm{ce}}$. Panel (c) displays plasma wave tracker data, which has a fine-frequency resolution over a limited bandwidth. The frequency axis is from 400 to $420 \mathrm{kHz}$, with a $32 \mathrm{~Hz}$ resolution.

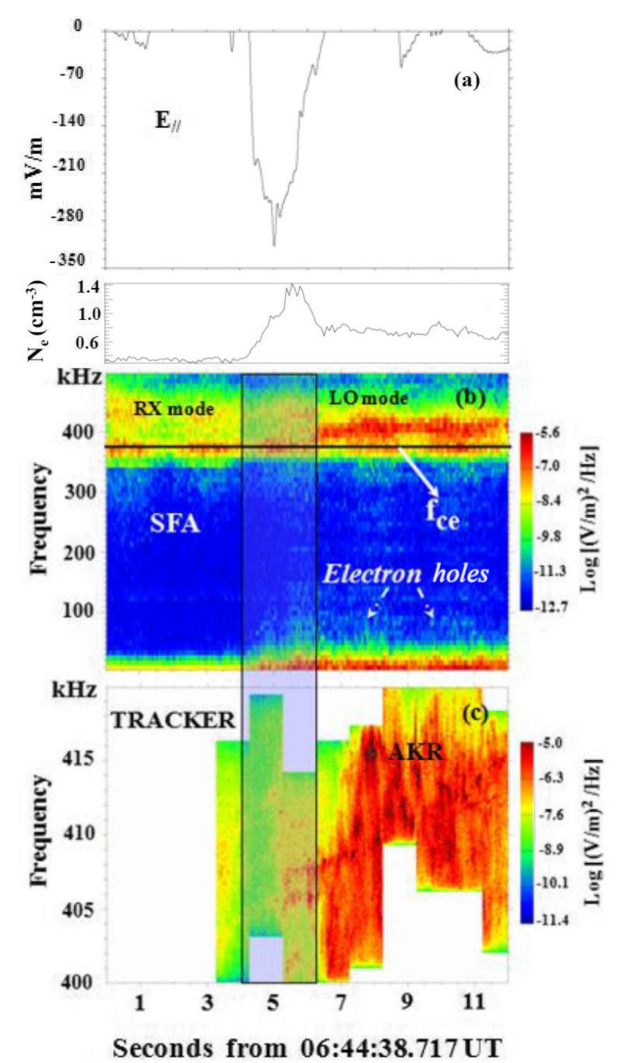

Figure 1. FAST spacecraft observations during the crossing of a localized double layer. The time duration is $12 \mathrm{~s}$. Panel (a) plots the parallel component of the anti-earthward-directed DC electric field together with the density variation. Panel (b) shows the electric power density as a function of frequency. The on-board sweep frequency analyser spans 0 to $500 \mathrm{kHz}$, with a $4 \mathrm{kHz}$ bandwidth. The dark horizontal line is the non-relativistic electron gyrofrequency $f_{\text {ce. }}$ Panel (c) reveals the presence of large-amplitude fine spectral AKR features measured by the plasma tracker. The frequency axis is from 400 to $420 \mathrm{kHz}$, with a $32 \mathrm{~Hz}$ resolution. In (b, c) the shaded blue region is correlated with the crossing of the parallel electric field.

From 06:44:38.717 UT as illustrated in Fig. 1a, according to the electron flux measurements, the FAST spacecraft stays in a region of depressed density $\left(\sim 0.3 \mathrm{~cm}^{-3}\right)$ during $\sim 3 \mathrm{~s}$. In the time interval $3-6 \mathrm{~s}$ the spacecraft enters a region of sharp density gradient. The density is substantially increased (roughly by a factor 5) and reaches $\sim 1.4 \mathrm{~cm}^{-3}$ in agreement with the cut-off frequency of the hiss emissions (Fig. 1b); then it decreases to $0.8 \mathrm{~cm}^{-3}$ and stays more or less constant during the rest of the selected time sequence. In accordance with the previous observations from the Polar spacecraft (Hull et al., 2003), the recorded large-amplitude parallel electric field takes place at the boundary separating high- and low-density plasma; this is probably the result of an ambipolar response of the plasma.

The power displayed in Fig. 1b was measured thanks to an electric antenna located in the satellite spin plane which 
contains the magnetic field. This antenna rotates at spacecraft spin period ( $5 \mathrm{~s}$ ) and alternately measures the parallel and perpendicular components of the generated AKR electric fields. It can be seen that, in the region of depressed density, the AKR emissions occurring near $f_{\text {ce }}$ are modulated at twice the spacecraft spin period. This modulation is such that the largest amplitudes of the recorded electric fields take place when the antenna is located in the direction perpendicular to the local magnetic field; only very weak amplitudes are registered in the parallel direction. This indicates that, in the density cavity, the wave electric field is polarized perpendicular to the ambient magnetic field, which implies that the radiation is generated in the right-hand circularly polarized extraordinary (RX) mode (Ergun et al., 1998). In contrast, in the enhanced-density region, the recorded AKR electric field displays a significant parallel component, which denotes that part of the radiation is generated in the LO mode.

The most striking feature in Fig. $1 \mathrm{~b}$ occurs from $\sim 06: 44: 45$ UT, in connection with the AKR LO-mode generation, when powerful AKR emissions are recorded at frequencies located significantly $(\sim 10 \%)$ above the local electron gyrofrequency $\left(f_{\text {ce }} \sim 365 \mathrm{kHz}\right)$. The AKR power associated with this event reaches $\sim 10^{-6}(\mathrm{~V} / \mathrm{m})^{2} / \mathrm{Hz}$; almost 2 orders of magnitude greater than the one measured in the cavity.

As illustrated in Fig. 1c, in association with the powerful AKR emissions which take place on the high-potential side of the DL, the FAST tracker detects AKR fine spectral features. These small-scale radiators appear as quasi-vertical structures with several hundred hertz bandwidths; they exhibit predominantly positive frequency drifts $(\mathrm{d} f / \mathrm{d} t>0)$ implying that the radiating structures propagate earthward.

Figure 2 reveals a $10 \mathrm{~ms}$ time sequence of the parallel electric field waveform recorded during the AKR elementary radiators generation. One distinguishes a series of largeamplitude $\left(\sim 120 \mathrm{mV} \mathrm{m}^{-1}\right)$ bipolar electric field structures which characterize the presence of electron holes (EHs); each of them lasts for about $150 \mu \mathrm{s}$. These nonlinear structures have a spatial extend of a few Debye lengths and are associated with a localized positive potential (Schamel, 1979). They have the polarity of a positive potential pulse. In the reference frame we are using, a positive value of the associated parallel electric field corresponds to the earthward direction which coincides with the beam propagation. It can be seen that in the present case, EHs always have a parallel electric field first directed earthward then anti-earthward; such a polarity indicates that they are indeed travelling in the electron beam direction. The present observations are consistent with the results of numerical simulations which show that EHs are generated on the high-potential side of a DL; they have a velocity of the order of the drift velocity of the associated electron beam (Newman et al., 2001; Goldman et al., 2003). All through the end of the selected time sequence from $\sim 5$ to $12 \mathrm{~s}$, the EHs appear in the spectrogram measured by the SFA as brief emissions of broadband noise (see Fig. 1b); they are

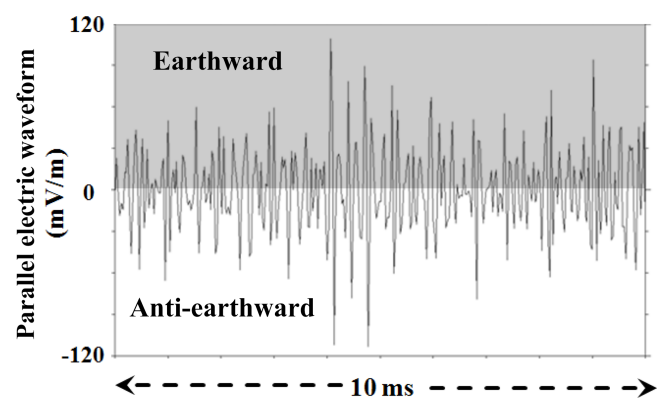

Figure 2. A $10 \mathrm{~ms}$ sequence of the fluctuations observed in the $32 \mathrm{kHz}$ quasi-parallel electric field waveform. The data were acquired on the high-potential side of the recorded DL at $\sim 06: 44: 45$ UT. They characterize the presence of EHs which have time spans of about $150 \mu$ s with amplitudes of $\sim 120 \mathrm{mV} \mathrm{m}^{-1}$. All the EHs have a positive then a negative polarity indicating that they are travelling earthward.

associated with fine AKR spectral features detected by the tracker.

Figure $3 \mathrm{a}$ and $\mathrm{b}$ display two electron distributions recorded during the selected time sequence; they are averaged on $0.8 \mathrm{~s}$. The horizontal axis is the velocity parallel to the magnetic field; the vertical axis is the perpendicular velocity. At 06:44:42 UT, inside the density cavity, the distribution illustrated in Fig. 3a exhibits a well-defined horseshoe shape and is associated with AKR emissions recorded at (and slightly below) $f_{\text {ce }}$. This distribution exhibits a positive slope, $\partial F_{\mathrm{e}}\left(v_{/ /}, v_{\perp}\right) / \partial v_{\perp}>0$, in a large pitch angle range and provides the free energy for AKR generation via the cyclotron maser instability (Ergun et al., 2000; Mutel et al., 2007); the excited waves propagate in the RX mode.

Figure $3 \mathrm{~b}$ reveals the electron distribution recorded at 06:44:45 UT in the regions of enhanced density located on the high-potential side of the recorded DL; it exhibits a pronounced beam-like character. The peak parallel energy of the electron beam reaches $\sim 14 \mathrm{keV}$; it is $\sim 7 \mathrm{keV}$ larger than in the cavity. The recorded electron distribution can be reproduced assuming that the electrons which form the horseshoe distribution, previously shown in Fig. 3a, have passed through a localized parallel potential drop of $\sim 7 \mathrm{keV}$. It can be seen that the highest energetic electrons form an incomplete ring in the pitch angle range $\theta \sim \pm 45^{\circ}$. At the same time, an anti-earthward parallel electric field component is measured to be of $\sim 300 \mathrm{mV} \mathrm{m}^{-1}$, implying the crossing of an accelerating structure with a parallel extension of $\sim 25 \mathrm{~km}$ which supports the potential drop. In these regions powerful recorded AKR emissions occur significantly above $f_{\text {ce }}$ (see Fig. 1b). 

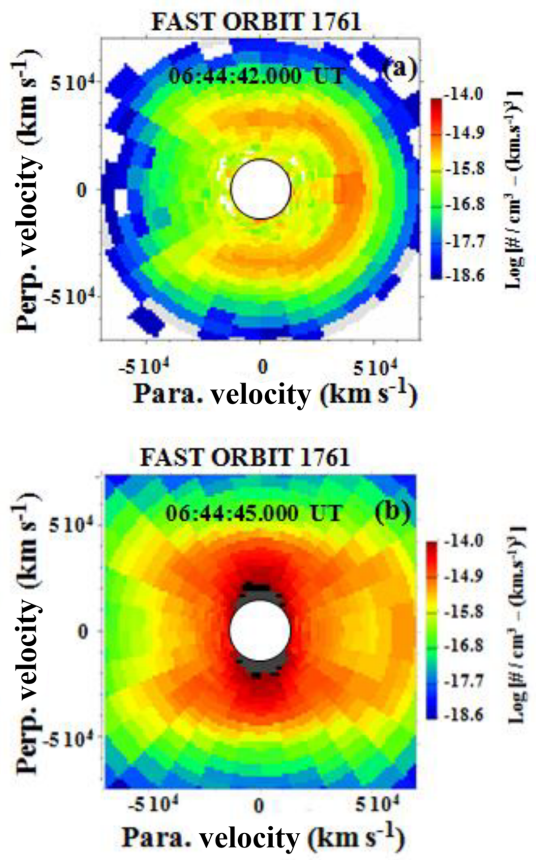

Figure 3. Panel (a) plots the full-ring horseshoe electron distribution recorded inside the density cavity at $\sim 06: 44: 42$ UT before the DL crossing. Panel (b) displays the electron distribution measured at $06: 44: 45 \mathrm{UT}$ in the regions of enhanced density located on the high-potential side of the recorded DL; it exhibits a pronounced beam-like character. The distributions are plotted in perpendicular, parallel velocity space. Positive values of the parallel velocities correspond to earthward propagation. Both distributions are averaged over $\sim 0.8 \mathrm{~s}$. The background electron fluxes with energy less than $\sim 1 \mathrm{keV}$ have been disregarded.

\section{Discussion}

\subsection{Free energy for AKR generation}

It is worth remembering that in the theory of the electron maser emission the gradient relevant to the generation of the radiation is the partial perpendicular velocity gradient on the electron distribution function. It can be expressed as the sum of two terms, namely

$$
\begin{aligned}
\partial F_{\mathrm{e}}\left(v_{/ /}, v_{\perp}\right) / \partial v_{\perp} & =\sin \theta \frac{\partial F_{\mathrm{e}}\left(v_{/ /}, v_{\perp}\right)}{\partial v} \\
& +\frac{\cos \theta}{v} \frac{\partial F_{\mathrm{e}}\left(v_{/ /}, v_{\perp}\right)}{\partial \theta}
\end{aligned}
$$

The first one is the derivative with respect to the electron velocity $v$; the second is the derivative with respect to the electron pitch angle $\theta$.

The present observations provide interesting information about the characteristics of electron distributions which provide free energy for AKR generation. Two main generation regions are identified:
- Inside the density, cavity the presence of a horseshoe electron distribution supplies the free energy for AKR generation. Because of the symmetry of this distribution function, the derivative $\partial / \partial \theta \approx 0$ with respect to $\theta$ can be neglected, resulting in $\partial F_{\mathrm{e}}\left(v_{/ /}, v_{\perp}\right) / \partial v_{\perp} \approx$ $\sin \theta \partial F_{\mathrm{e}}\left(v_{/ /}, v_{\perp}\right) / \partial v$. The dominant emission is the RX mode; it is recorded at frequencies in the neighbourhood of $f_{\text {ce. }}$. This is in agreement with previous observations (Louarn et al., 1990; Ergun et al., 2000).

- At the cavity interface, where sharp density gradients are located, large-amplitude AKR emissions are recorded in the presence of a DL; they take place on the high-potential side of the DL. The free energy is supplied by an electron distribution exhibiting perpendicular velocity gradients induced by the presence of an incomplete ring of energetic electrons together with the existence of a lower-energy tail at localized pitch angles; in these circumstances both terms in Eq. (1) must be taken into account. The recorded AKR emissions occur at frequencies located significantly above $f_{\text {ce }}$, and the polarization of the waves indicates the presence of a significant power in the LO-mode radiation.

\subsection{Radiation from electron holes}

The present experimental observations show that bandlimited radiation above $f_{\text {ce }}$ takes place in the presence of EHs, which may distort the electron distribution and therefore modulate the electron cyclotron instability (Pottelette et al., 2001; Treumann et al., 2011).

In the auroral acceleration region the typical spatial extension of the $\mathrm{EH}$ parallel to the magnetic field amounts to $L_{/ /} \sim 1 \mathrm{~km}$ (several Debye lengths). Observations from the POLAR spacecraft indicate that, when the plasma frequency is small as compared to the electron gyrofrequency, EHs are rather spherically symmetric (Franz et al., 2000); a conclusion which has also been drawn by Berthomier et al. (2003) from simplified magnetized theory in a strong magnetic field. Having a perpendicular size $L_{\perp} \approx L_{/ /}$, an EH is well-suited for acting as an efficient radiating antenna in the kilometre wavelength range. At FAST altitudes such a radial size is well suited to the generation of AKR elementary events characterized by a $\Delta f \sim 100 \mathrm{~Hz}$ bandwidth (Pottelette and Pickett, 2007).

Numerical simulations demonstrate that EHs occupy a substantial part of velocity space while being very narrow in configuration space (Newman et al., 2001; Goldman et al., 2003). Due to the finite gyroradius of the trapped electrons, any EHs generated in magnetized plasmas will necessarily have a finite extension in perpendicular velocity space. This extension gives rise to perpendicular velocity space gradients on the electron distribution function which are confined to the edges of the EHs in parallel and perpendicular direction (Treumann et al., 2011). The radiation generated from 
the outer boundary of the hole can be powerful if the localized phase space gradients are sharp enough. Unfortunately these gradients cannot be experimentally quantified because the characteristic transit time of EHs across the spacecraft antenna lasts for a few hundred microseconds. One would need to measure the electron distribution, say, from $10 \mathrm{eV}$ to $20 \mathrm{keV}$ on the same timescale. Clearly, for the time being, an instrumentation capable of this cannot be based on a spacecraft.

In terms of the electron velocity components, the resonance condition, for mildly relativistic electrons satisfying the cyclotron instability, is fulfilled around the following resonant circle (Wu and Lee, 1979):

$\left(v_{/ /}-v_{\mathrm{a}}\right)^{2}+v_{\perp}^{2}=v_{\mathrm{r}}^{2}$,

where $v_{\mathrm{a}}$ is the centre of the circle in the direction parallel to the magnetic field

$v_{\mathrm{a}}=\frac{k_{/ / c^{2}}}{\omega_{\mathrm{ce}}}$

while the radius is

$v_{\mathrm{r}}=\sqrt{v_{\mathrm{a}}^{2}+2 c^{2}\left(1-\omega / \omega_{\mathrm{ce}}\right)}$.

In both equations $\omega$ is the angular wave frequency, $\omega_{\text {ce }}$ the angular electron gyrofrequency, $k_{/ /}$the wave vector parallel to the magnetic field, and $c$ the speed of light.

In order to satisfy the general weakly relativistic resonance condition for the maser instability, the velocity $V_{\mathrm{h}}$ of the hole must be such that the perpendicular gradients localized at the hole coincide with the resonance contour given by (2), which implies $V_{\mathrm{h}}=V_{\mathrm{a}}$. This equality provides a determination of the parallel wave number through the $\mathrm{EH}$ velocity

$k_{/ /}=\frac{\omega_{\mathrm{ce}} V_{\mathrm{h}}}{c^{2}}$

The above parallel wave number does not vanish as long as $V_{\mathrm{h}} \neq 0$. In the observer frame the radiation emitted by an $\mathrm{EH}$ is oblique, while in the electron hole reference frame (where $V_{\mathrm{h}}$ and consequently $k_{/ /}$are 0 ) the radiation is predominantly generated in a perpendicular direction and occurs in a frequency range located at or slightly beneath the local nonrelativistic electron-cyclotron frequency $f_{\text {ce }}$. As such nonlinear structures propagate earthward, at a velocity of the order of the electron beam velocity, a satellite located below the altitude of an accelerating DL will mainly observe radiation from incoming holes. In these circumstances, the change in frequency due to the displacement of an EH along the magnetic field is described by the relativistic Doppler effect which, for the observed frequency of the radiation, results in

$f=f_{\mathrm{ce}} \frac{\sqrt{1-\beta_{\mathrm{h}}^{2}}}{1-\beta_{\mathrm{h}}}$,

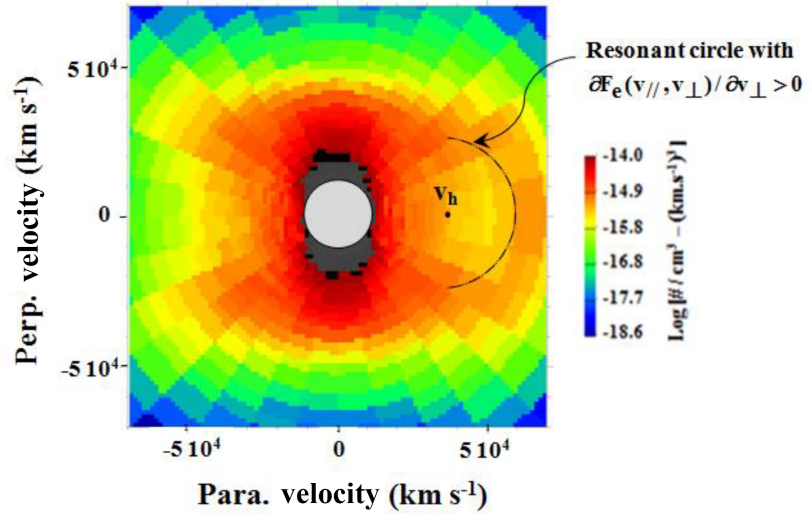

Figure 4. Enlargement of Fig. 3b illustrating the integration surface in the EH stationary frame. The centre of the resonant circle (dashed line) is at parallel velocity $V_{\mathrm{h}}$, equal to half the beam velocity. Only the part of this circle which passes the positive perpendicular velocity space gradient contributes to a positive growth rate for the cyclotron maser instability; it is limited to $-45^{\circ}<\theta<45^{\circ}$.

where $\beta_{\mathrm{h}}=V_{\mathrm{h}} / c$. Fig. 1c shows that, in the presence of EHs, the excited AKR frequency range is $\sim 410 \pm 10 \mathrm{kHz}$. The local electron gyrofrequency is $f_{\text {ce }} \sim 365 \mathrm{kHz}$; the use of Eq. (6) leads to $V_{\mathrm{h}} \sim(35000 \pm 5000) \mathrm{km} \mathrm{s}^{-1}$. This is almost half the value of the beam velocity (see Fig. 3b). Such a velocity range is in excellent agreement with the electronelectron two-stream instability as the generation mechanism of these nonlinear structures (Treumann and Baumjohann, 1997). It can be seen in Fig. 3b that the drift velocity of the propagating electron beam is much larger than the electron thermal stream; this induces an appreciable growth rate of this instability.

As previously emphasized, radiation generation via the cyclotron maser implies the production of a steep positive velocity gradient at the boundary of the EHs, particularly near the electron beam bounding this nonlinear structure from the high-speed side. EHs extend in configuration space in the perpendicular direction, at least over distances of the trapped electron gyro radius. The presence of these trapped electrons induces the required velocity space gradients in the perpendicular direction.

An example of an integration surface satisfying the requests $\partial F_{\mathrm{e}}\left(v_{/ /}, v_{\perp}\right) / \partial v_{\perp}>0$ is indicated by a dashed line in Fig. 4. This figure is an enlargement of Fig. 3b, which displays the two-dimensional electron distribution recorded on the high-potential side of the DL; the radius of the resonant circle is $V_{\mathrm{r}}=V_{\mathrm{b}}-V_{\mathrm{h}}$. 


\subsection{Electron cyclotron maser growth rate}

Using polar coordinates, the growth rates for the RX and LO modes can be respectively written as (Mutel et al., 2007)

$$
\left.\frac{\omega_{i}}{\omega_{\mathrm{ce}}}\right|_{\mathrm{RX}}=\left.\mu_{R} \frac{\left(\pi v_{\mathrm{r}} c\right)^{2}}{4 n_{\mathrm{e}}}\left(\frac{\omega_{\mathrm{pe}}}{\omega_{\mathrm{ce}}}\right)^{2} \int_{0}^{\pi} \mathrm{d} \theta \sin ^{2}(\theta) \frac{\partial F_{\mathrm{e}}(\boldsymbol{v})}{\partial v_{\perp}}\right|_{|\boldsymbol{v}|=v_{\mathrm{r}}}
$$

and

$$
\left.\frac{\omega_{i}}{\omega_{\mathrm{ce}}}\right|_{\mathrm{LO}}=\left.\frac{\pi^{2} v_{\mathrm{r}}^{4}}{16 n_{\mathrm{e}}}\left(\frac{\omega_{\mathrm{pe}}}{\omega_{\mathrm{ce}}}\right)^{2} \int_{0}^{\pi} \mathrm{d} \theta \sin ^{2}(2 \theta) \frac{\partial F_{\mathrm{e}}(\boldsymbol{v})}{\partial v_{\perp}}\right|_{|\boldsymbol{v}|=v_{\mathrm{r}}},
$$

where $n_{\mathrm{e}}$ is the number density of energetic electrons and $\omega_{\text {pe }}$ is the angular plasma frequency. The integrals must be solved accounting for resonance along the resonant circle in velocity space defined by Eq. (2); only the part of this circle which passes the positive perpendicular velocity space gradient contributes to a positive growth rate. The dimensionless factor $\mu_{R} \sim 0.14$ corrects for the effect of the plasma dispersion. Note that the dependence of the growth rate on the velocity distribution appears as a linear function of the perpendicular derivative weighted by a simple geometrical factor centred at $90^{\circ}$ (RX mode) or 45 and $135^{\circ}$ (LO mode). Without the influence of this geometrical factor, the growth rate for the ordinary mode is smaller than that of the extraordinary mode by $\mathrm{O}\left(v_{\mathrm{r}}^{2} / \mu_{R} c^{2}\right)$.

Using Eqs. (7) and (8), it can be shown that in the auroral cavity, characterized by the presence of a horseshoe distribution of the type illustrated in Fig. 3a, the RX to LO growth rate ratio is $\sim 10^{-3}$ (Mutel et al., 2007). In the cavity, the resonant circle which encompasses the largest velocity gradients is located at the origin of the velocity space of Fig. 3a (Ergun et al., 2000); its radius is $V_{\mathrm{r}}=c \sqrt{2\left(1-f / f_{\mathrm{ce}}\right)}$. The recorded polarization of the radiation is consistent with $\mathrm{X}$ mode generation at a frequency located slightly below $f_{\text {ce }}$; it requires $k_{/ /}=0$ in order to fulfill Eq. (2).

As emphasized earlier, in order to quantify the emission of radiation generated by an $\mathrm{EH}$, it is useful to work in the reference frame of the hole which is not centred at the origin of the velocity space. Given the order of magnitude of the velocity range of the EHs recorded at the edge of the cavity, a resonant circle which satisfies the condition for strong maser emission is plotted with a dashed line in Fig. 4. Only the part of this circle which passes the positive perpendicular velocity space gradient contributes to a positive growth rate; it is limited to $-45^{\circ}<\theta<45^{\circ}$. As seen in Eqs. (7) and (8), the growth rates of the RX and LO modes are a sensitive function of $\sin ^{2}(\theta)$ and $\sin ^{2}(2 \theta)$, respectively. Given the integration interval restricted to $-45^{\circ}<\theta<45^{\circ}$, the value of the geometrical factor is much larger for the LO mode than for the RX mode. This may explain the significant power recorded in the LO at the edges of the auroral density cavity which is sometimes observed (Louarn, 2006).

\section{Conclusions}

The present paper provides new additional evidence related to the generation of elementary-scale radiation by the EHs.

There is undeniable cross-fertilization between laboratory and space experiments regarding radiation generation. The observations performed in the AKR source region strengthen those previously acquired from laboratory experiments (Gunell and Löfgren, 1997; Brenning et al., 2004). Actually, part of the AKR radiation appears to be generated thanks to the existence of nonlinear structures, located on the high-potential side of a DL, which act as a sender antenna. Such localized large-amplitude parallel electric fields are recorded inside high-density gradients located at the interface of the auroral cavity. In agreement with previous studies (Pottelette et al., 2001, 2014; Treumann et al., 2011), the nonlinear structures which give rise to the fine structure in the AKR spectrum can be identified as electron holes. A recent theoretical study calculating the AKR growth rates in the presence of EHs embedded in the electron distribution supports this interpretation (Zhao et al., 2016).

In the hole frame, the radiation is excited near the local gyrofrequency, while, in the satellite frame, the excited frequency range appears to be shifted up because of the Doppler effect generated by approaching holes (Treumann et al., 2011). The use of the measurement of the frequency shift allows the determination of the EHs velocity; it turns out that these nonlinear structures move at about half the value of the beam velocity. Such a velocity range is in excellent agreement with the electron-electron two-stream instability and implies that the EHs are caused by the nonlinear evolution of the electrostatic waves generated by this instability. This is a very fundamental result which shows that the generation mechanism of EHs can be derived from the characteristics of the radiation they transmit.

The radiation emitted by an EH has a finite $k_{/ /}$and can consequently escape from the source region. Regarding the AKR polarization, it has also been underlined that, on the high-potential side of a DL, the shape of the electron distribution notably enhances the growth rate of the LO mode as compared to its value in the density cavity.

As EHs seem to be strong sources of non-thermal radio radiation from the aurora, all these observations suggest that they should play an important role in other magnetized planetary objects and more generally in astrophysical plasmas. The formation of EHs and their associated radiation mechanism are highly nonlinear and cannot be accessed by a perturbation approach. The only way of investigating such processes is through numerical simulations. For the time being, it is generally believed that the short spatial-scale perpendicular velocity gradient at the hole boundary, generated by the presence of trapped electrons, is steep and contributes to the electron cyclotron maser. However, the migration of the EHs in velocity space, as the electron velocity distribution function evolves from a beam near the high-potential side of the 
DL, is crucial to the understanding of the role of these nonlinear structures as small-scale radiators. Unfortunately, in the presence of the converging Earth's magnetic field, the evolution of an EH, including its structure, stability (life time) and propagation speed, remain essentially unexplored by numerical simulations.

Data availability. The data are publicly accessible at the following address: http://sprg.ssl.berkeley.edu/fast/scienceprod/welcome. html.

Competing interests. The authors declare that they have no conflict of interest.

Acknowledgements. The FAST mission is a project of the Space Sciences Laboratory of the University of California at Berkeley run under the auspices of NASA. The authors are indebted to Charles W. Carlson and Robert E. Ergun for providing particle and wave data as well as for some useful discussions. This research has been initiated within the France-Berkeley program.

The topical editor, Elias Roussos, thanks two anonymous referees for help in evaluating this paper.

\section{References}

Benediktov, E. A., Getmantsev, C. G., Sazonoy, Y. A., and Tarasov, A. F.: Preliminary results of measurement of the intensity of distributed extraterrestrial radio-frequency emission at 725 and $1525-\mathrm{kHZ}$ frequencies by the satellite elektron-2, Kosm. Issled., 3, 614-618, 1965.

Berthomier, M., Pottelette, R., Muschietti L., Roth, I., and Carlson, C. W.: Scaling of three dimensional electron phase space density holes observed by FAST in the auroral downward current region, Geophys. Res. Lett., 30, 2148-152, https://doi.org/10.1029/2003GL018491, 2003.

Brenning, N., Koepke, M. E., Axnäs, I., and Raadu, M. A.: Electromagnetic radiation from double layers, 12th International Congress on Plasma Physics, 25-29 October, Nice, France, 2004.

Chiu, Y. T. and Schulz, M.: Self-consistent particle and parallel electrostatic field distributions in the magnetosphericionopheric auroral region, J. Geophys. Res., 83, 629-642, https://doi.org/10.1029/JA083iA02p00629, 1978.

Ergun, R. E., Carlson, C. W., McFadden, J. P., Mozer, F. S., Delory, G. T., Peria, W., Chaston, C., Temerin, M., Elphic, R., Strangeway, R. J., Pfaff, R., Cattel, C. A., Klumpar, D., Shelly, E., Peterson, W., Moebius, E., and Kistler, L.: FAST satellite wave observations in the AKR source region, Geophys. Res. Lett., 25, 2061-2064, https://doi.org/10.1029/98GL00570, 1998.

Ergun, R. E., Carlson, C. W., McFadden, J. P., Delory, G. T., Strangeway, R. J., and Pritchett, P. L.: Electron-Cyclotron Maser Driven by Charged-Particle Acceleration from Magnetic Field-aligned Electric Fields, Astrophys J., 538, 456, https://doi.org/10.1086/309094, 2000.
Franz, J. R., Kintner, P. M., Seyler, C. E., Pickett, J. S., and Scudder, J. D.: On the perpendicular scale of electron phase-space holes, Geophys. Res. Lett., 27, 169-173, https://doi.org/10.1029/1999GL010733, 2000.

Goldman, M. V., Newman, D. L., and Ergun, R. E.: Phase-space holes due to electron and ion beams accelerated by a currentdriven potential ramp, Nonlin. Processes Geophys., 10, 37-44, https://doi.org/10.5194/npg-10-37-2003, 2003.

Gunell, H. and Löfgren, T.: Electric field spikes formed by electron beam-plasma interaction in plasma density gradients, Phys. Plasma, 4, 2805, https://doi.org/10.1063/1.872413, 1997.

Gurnett, D. A.: The earth as a radio source - Terrestrial kilometric radiation, J. Geophys. Res., 79, 4227-4238, https://doi.org/10.1029/JA079i028p04227, 1974.

Hull, A. J., Bonnell, J. W., Mozer, F. S., Scudder, J. D., and Chaston, C. C.: Large parallel electric fields in the upward current region of the aurora: Evidence for ambipolar effects, J. Geophys. Res., 108, 1265, https://doi.org/10.1029/2002JA009682, 2003.

Lindberg, L.: Observations of Electromagnetic Radiation from Plasma in Presence of a Double Layer, Phys. Scripta, 47, 92-95, https://doi.org/10.1088/0031-8949/47/1/016, 1993.

Löfgren, L. and Gunell, H.: Interacting eigenmodes of a plasma diode with a density gradient, Phys. Plasmas, 5, 590, https://doi.org/10.1063/1.872751, 1998.

Louarn, P.: Generation of auroral kilometric radiation in bounded source regions, in Geospace Electromagnetic Waves and Radiation, Lecture Notes in Physics (LNP), Vol. 687, Springer-Verlag, Berlin-Heidelberg-New York, 55-86, 2006.

Louarn, P., Roux, A., De Feraudy, H., Le Quéau, D., André, M., and Matson, L.: Trapped electrons as a free energy source for the auroral kilometric radiation, J. Geophys. Res., 95, 5983, https://doi.org/10.1029/JA095iA05p05983, 1990.

Mutel, R. L., Peterson, W. M., Jaeger, T. R., and Scudder J. D.: Dependence of cyclotron maser instability on electron velocity distributions and perturbations by solitary waves, J. Geophys. Res., 112, A07211, https://doi.org/10.1029/2007JA012442, 2007.

Newman, D. L., Goldman, M. V., Ergun, R. E., and Mangeney, A.: Formation of Double Layers and Electron Holes in a Current-Driven Space Plasma, Phys. Rev. Lett., 87, 255001, https://doi.org/10.1103/PhysRevLett.87.255001, 2001.

Persoon, A. M., Gurnett, D. A., Peterson, W. K., Waite, Jr., J. H., Burch, J. L., and Green, J. L.: Electron density depletion in the nightside auroral zone, J. Geophys. Res., 93, 1871, https://doi.org/10.1029/JA093iA03p01871, 1988.

Pottelette, R. and Pickett, J.: Phase space holes and elementary radiation events, Nonlin. Processes Geophys., 14, 735-742, https://doi.org/10.5194/npg-14-735-2007, 2007.

Pottelette, R., Treumann, R. A., and Berthomier, M.: Auroral Plasma Turbulence and the cause of AKR fine structure, J. Geophys. Res., 106, 8465-8476, https://doi.org/10.1029/2000JA000098, 2001.

Pottelette, R., Berthomier, M., and Pickett, J.: Radiation in the neighbourhood of a double layer, Ann. Geophys., 32, 677-687, https://doi.org/10.5194/angeo-32-677-2014, 2014.

Schamel, H.: Theory of electron holes, Phys. Scripta, 20, 336-342, https://doi.org/10.1088/0031-8949/20/3-4/006, 1979.

Treumann, R. A.: The electron-cyclotron maser for astrophysical application, Astron. Astrophys. Rev., 13, 229-315, https://doi.org/10.1007/s00159-006-0001-y, 2006. 
Treumann, R. A. and Baumjohann, W.: Advanced Space Plasma Physics, Imperial College Press, London, 1997.

Treumann, R. A., Baumjohann, W., and Pottelette, R.: Electron-cylotron maser radiation from electron holes: upward current region, Ann. Geophys., 29, 1885-1904, https://doi.org/10.5194/angeo-29-1885-2011, 2011.

Volwerk, M.: Radiation from electrostatic double layers in laboratory plasmas, J. Phys. D Appl. Phys., 26, 1192-1202, https://doi.org/10.1088/0022-3727/26/8/007, 1993.
Wu, C. S. and Lee, L. C.: A theory of the terrestrial kilometric radiation, Astrophys. J., 230, 621-626, https://doi.org/10.1086/157120, 1979.

Zhao, G. Q., Chu, Y. H., Feng, H. Q., and Wu, D. J.: The effect of electron holes on cyclotron maser emission driven by horseshoe distributions, Phys. Plasmas, 23, 114505, https://doi.org/10.1063/1.4968220, 2016. 